\title{
Therapeutic Effect Analysis of Sodium Aescinate Tablets on Knee Osteoarthritis Combined with Synovitis
}

\author{
Xueqin Zeng, Baohui Wang, Liang Li, Tao Lei, Huajian Liu, Yindi Sun* \\ TCM Orthopaedic Pain Ward of Rehabilitation Hospital, Hong Hui Hospital, Xi'an Jiaotong University, Xi'an 710054,
} Shaanxi Province, China

*Corresponding author: Yindi Sun, 1158122013@qq.com

\begin{abstract}
Objective: To observe therapeutic effect of sodium aescinate Tablets on knee osteoarthritis complicated with synovitis. Methods: 86 patients with knee osteoarthritis combined with synovitis treated in the Honghui Hospital Affiliated to Xi'an Jiaotong University from January 2018 to December 2019 were randomly divided into control group and intervention group, with 43 cases in each group. The control group was given routine treatment, and the intervention group was given sodium aescinate Tablets orally on the basis of routine treatment, twice a day, 2 tablets each time. The curative effect was evaluated after 2 weeks of treatment. The knee pain and joint function of the two groups were evaluated by Visual Analogue Scale (VAS) and Western Ontario and McMaster Universities Arthritis Index (WOMAC), and the clinical effects of the two groups were compared. Results: Before treatment, there was no significant difference in general information, VAS and WOMAC scores between the two groups $(\mathrm{P}>0.05)$. After treatment, the VAS and WOMAC scores of the two groups were both lower than those before treatment, and the difference was statistically significant $(\mathrm{P}<0.05)$. The vas and WOMAC scores of the intervention group were lower than those of the control group (VAS, $1.87 \pm 0.79$ vs $3.38 \pm 0.81$. In this study, sodium aescinate tablets were applied to the treatment of KOA combined with synovitis. The results showed that the intervention group was better than the control group in alleviating knee pain, improving joint function and improving clinical efficacy, and the difference was statistically significant. The total effective rate of the intervention group was higher than that of the control group $(100 \%$ vs $86.05 \%)$, and the difference was statistically significant $(P<0.05)$. Conclusion: Sodium aescinate tablet in the treatment of knee osteoarthritis combined with synovitis can effectively alleviate knee pain, improve joint function and improve clinical efficacy.
\end{abstract}

Keywords: Knee osteoarthritis; Synovitis; Pain; Knee function; Quality of life

Publication date: November 2021; Online publication: November 30, 2021

\section{Introduction}

Knee osteoarthritis (KOA) is a chronic and degenerative disease characterized by degeneration and destruction of knee cartilage and hyperosteogeny around and under cartilage ${ }^{[1]}$. The main clinical features are joint pain, swelling, stiffness and decreased mobility, which seriously affect the quality of life of patients ${ }^{[2]}$. Studies have shown that the prevalence of KOA in Chinese population is $8.1 \%$, and the prevalence increases with age ${ }^{[3]}$. Knee synovitis is mostly secondary to KOA, mainly due to mechanical biochemical stimulation caused by cartilage degeneration and bone hyperplasia, leading to knee joint synovial edema, exudation and fluid accumulation. KOA is often combined with knee synovitis. Therefore, clinicians should take measures to prevent and treat synovitis while treating KOA. Sodium aescinate is a kind of triterpenoid saponin sodium salt isolated and extracted from Aesculus chinensis fruit. It shows certain effects in 
scavenging free radicals, anti-permeability, improving blood circulation and stabilizing vascular endothelial cells ${ }^{[4-5]}$. In view of this, this study intends to reveal the effect of sodium aescinate tablets on knee osteoarthritis combined with synovitis through clinical control study.

\section{Materials and methods}

\subsection{General information}

86 patients with KOA combined with synovitis hospitalized in the rehabilitation hospital of the Honghui Hospital Affiliated to Xi'an Jiaotong University from January 2018 to December 2019 were selected, including 37 males and 49 females, aged from 31 to 82 years, with an average age of (58.76 \pm 13.34$)$ years, a course of 6 months to 10 years, and an average course of $(42.58 \pm 21.95)$ months.

\subsubsection{Inclusion criteria}

(1) It meets the diagnostic criteria of $\mathrm{KOA}$ in the guidelines for the diagnosis and treatment of osteoarthritis (2018 Edition) ${ }^{[6]}$.

(2) It meets the diagnostic criteria of synovitis in Orthopedics Tendon Disease ${ }^{[7]}$, such as: history of chronic injury stimulation, joint swelling, positive floating patella test, and synovial diseases such as intra-articular hematoma, synovial tuberculosis, chorionic pigmented nodular synovitis and synovial tumor are excluded.

(3) Patients who are suitable for non-surgical treatment; Patients are willing to participate in the research and signed informed consent.

\subsubsection{Exclusion criteria}

(1) Patients are combined with severe heart, cerebrovascular, liver, kidney and other organ diseases.

(2) Patients are combined with other diseases which may affect knee pain and swelling.

(3) Patients with repeated massive effusion in knee joint cavity and are clinically classified as severe osteoarthritis.

(4) Pregnant or lactating women.

(5) Patients failed to take prescribed medications or to take other related medications or receive other related treatments during treatment.

The study has been approved by the hospital medical ethics committee. The patients were randomly divided into control group and intervention group, with 43 cases in each group. There was no significant difference between the two groups in gender, age, course of disease, location of disease and body mass index (BMI) $(P>0.05)$. See Table 1.

Table 1. Patients' information in two groups (n / $\bar{x} \pm s)$

\begin{tabular}{lcccccc}
\hline \multicolumn{1}{c}{ Group } & $\begin{array}{c}\text { Number } \\
\text { of cases }\end{array}$ & $\begin{array}{c}\text { Gender } \\
\text { (e.g., male } / \\
\text { female) }\end{array}$ & Age (years) & $\begin{array}{c}\text { Course of } \\
\text { disease (years) }\end{array}$ & $\begin{array}{c}\text { Diseased parts } \\
\text { (example, left / } \\
\text { right / bilateral) }\end{array}$ & BMI \\
\hline $\begin{array}{l}\text { Control } \\
\text { group }\end{array}$ & 43 & $18 / 25$ & $59.06 \pm 14.54$ & $41.15 \pm 21.01$ & $19 / 17 / 7$ & $24.24 \pm 3.01$ \\
$\begin{array}{l}\text { Intervention } \\
\text { group }\end{array}$ & 43 & $16 / 27$ & $58.79 \pm 13.73$ & $40.97 \pm 21.43$ & $18 / 19 / 6$ & $24.76 \pm 2.74$ \\
$x^{2} / t$ & & 0.195 & 0.768 & 0.651 & 0.215 & 0.462 \\
$P$ & & 0.659 & 0.434 & 0.493 & 0.898 & 0.633 \\
\hline
\end{tabular}




\subsection{Treatment method}

Patients in both groups were given celecoxib capsules orally (Pfizer, H20140106, 200mg * 6 capsules), once a day, 1 capsule each time; Ultrashort wave physiotherapy (slight heat, 15min / time, 2 times / D, 5 days / week) and massage therapy (25-30min / time, 1 time / D, 5 times / week). The intervention group was given sodium aescinate tablets orally (Shandong LVYE Pharmaceutical, H20051590, 30mg * 12 tablets), twice a day, 2 tablets each time. The curative effects of both groups were evaluated after 2 weeks of treatment.

\subsection{Observation index and curative effect evaluation}

\subsubsection{Visual analogue scale (VAS) ${ }^{[8]}$}

A $10 \mathrm{~cm}$ long line was drawn on white paper, and delimited by millimeter. One end of the line is marked with "no pain" (0 points), the other end is marked with "extremely painful" (10 points), and ask the patient to mark a point on the line according to the degree of knee joint pain, which is the pain score. The higher the score is, the more obvious the pain is.

\subsubsection{Western Ontario and McMaster Universities Arthritis Index (WOMAC) ${ }^{[9]}$}

This includes pain ( 5 items, 20 points), stiffness ( 2 items, 8 points) and knee function (17 items, 68 points), which is used to evaluate the structure and function of knee joint, with a total score range of $0 \sim 96$ points. The higher the score is, the worse the knee function is.

\subsubsection{The efficacy evaluation criteria refering to the criteria for judging the efficacy of diseases in} the treatment of osteoarthritis in "Guiding Principles for Clinical Research of New Chinese Medicine" (revised in 2002) ${ }^{[10]}$

Clinical control: The pain and other symptoms disappeared, the joint activity was normal, and the score reduction $\geq 95 \%$; Remarkable effect: The pain and other symptoms disappeared, the joint activity was normal, and 70\% $\leq$ score reduction $<95 \%$; Efficient: Pain and other symptoms were basically eliminated, joint activity was slightly limited, and $30 \% \leq$ score reduction $<70 \%$; Inefficient: There was no significant improvement in pain and joint activity, and the score reduction $<30 \%$. Total response rate $=($ clinical control + remarkable effect + efficient) $/$ total number of cases $\times 100 \%$.

\subsection{Statistical methods}

SPSS18.0 statistical software was used to process the data. The measurement data were expressed as $\overline{\mathrm{x}} \pm \mathrm{s}$, and t-test was used for comparison; The counting data were expressed in $\%$, the comparison was performed by $\chi^{2}$ test, and the difference was statistically significant $(\mathrm{P}<0.05)$.

\section{Results}

\subsection{Comparison of VAS and WOMAC scores between the two groups}

Before treatment, there was no significant difference in VAS and WOMAC scores between the two groups $(\mathrm{P}>0.05)$; After treatment, the VAS and WOMAC scores of the two groups were both lower than those before treatment, and the difference was statistically significant $(\mathrm{P}<0.05)$. VAS and WOMAC scores in the intervention group were lower than those in the control group, which is statistically significant $(\mathrm{P}<0.05)$, as shown in Table 2 and Table 3.

\subsection{Clinical efficacy}

The total effective rate in the intervention group was higher than that in the control group, and the difference was statistically significant $(\mathrm{P}<0.05)$, as shown in Table 4. 
Table 2. Comparison of VAS scores $[(\overline{\mathrm{x}} \pm \mathrm{s})$ points $]$

\begin{tabular}{llllll}
\hline Group & $\begin{array}{l}\text { Number } \\
\text { cases }\end{array}$ & $\begin{array}{l}\text { of } \\
\text { Before } \\
\text { treatment }\end{array}$ & $\begin{array}{l}\text { After } \\
\text { treatment }\end{array}$ & \#t value & \#P value \\
\hline Control group & 47 & $7.37 \pm 1.24$ & $3.38 \pm 0.81$ & 15.923 & $<0.001$ \\
Intervention group & 51 & $7.27 \pm 1.33$ & $1.87 \pm 0.79$ & 20.671 & $<0.001$ \\
"t value & & 0.343 & 7.922 & & \\
"P value & & 0.733 & $<0.001$ & & \\
\hline
\end{tabular}

Notes: VAS: Visual analog scale. ${ }^{*}$, ${ }^{*} \mathrm{P}$ : Comparison between groups; ${ }^{*}$, ${ }^{\#} \mathrm{P}$ : Intra-group comparison before and after treatment

Table 3. Comparison of WOMAC scores $[(\bar{x} \pm s)$ points]

\begin{tabular}{|c|c|c|c|c|}
\hline Group & $\begin{array}{l}\text { Control group }(n= \\
43)\end{array}$ & $\begin{array}{l}\text { Intervention } \\
\text { group }(n=43)\end{array}$ & t t value & *P value \\
\hline \multicolumn{5}{|c|}{ Before treatment } \\
\hline Pain & $14.89 \pm 2.39$ & $14.71 \pm 2.74$ & 0.279 & 0.781 \\
\hline Stiff & $5.71 \pm 1.30$ & $5.74 \pm 1.15$ & 0.098 & 0.922 \\
\hline Knee function & $46.49 \pm 6.81$ & $47.71 \pm 6.99$ & 0.745 & 0.459 \\
\hline Total score & $67.09 \pm 7.30$ & $68.17 \pm 7.63$ & 0.608 & 0.545 \\
\hline \multicolumn{5}{|l|}{ After treatment } \\
\hline Pain & $7.46 \pm 2.59^{\#}$ & $4.37 \pm 1.11^{\#}$ & 6.468 & $<0.001$ \\
\hline Stiff & $3.06 \pm 1.03^{\#}$ & $2.26 \pm 0.66^{\#}$ & 3.881 & $<0.001$ \\
\hline Knee function & $19.49 \pm 4.93^{\#}$ & $15.37 \pm 3.80^{\#}$ & 3.911 & $<0.001$ \\
\hline Total score & $30.16 \pm 6.11^{\#}$ & $22.03 \pm 3.81^{\#}$ & 6.574 & $<0.001$ \\
\hline
\end{tabular}

Notes: WOMAC: Western Ontario and McMaster Universities Arthritis Index. 't, *P: Comparison between groups; Comparison before and after treatment in the group, ${ }^{\#} \mathrm{P}<0.05$

Table 4. Comparison of clinical efficacy between the two groups [n (\%)]

\begin{tabular}{lllllll}
\hline Group & $\begin{array}{l}\text { Number } \\
\text { of cases }\end{array}$ & $\begin{array}{l}\text { Clinical } \\
\text { control }\end{array}$ & $\begin{array}{l}\text { Remarkable } \\
\text { effect }\end{array}$ & Effective & Inefficient & $\begin{array}{l}\text { Total effective } \\
\text { rate (\%) }\end{array}$ \\
\hline $\begin{array}{l}\text { Control group } \\
\text { Intervention }\end{array}$ & 43 & $9(20.93)$ & $13(30.23)$ & $15(34.89)$ & $6(13.95)$ & 86.05 \\
group & 43 & $17(39.53)$ & $19(44.19)$ & $7(16.28)$ & $0(0)$ & 100 \\
$x^{2}$ & & & & & & 4.479 \\
$P$ & & & & & & 0.34 \\
\hline
\end{tabular}

\section{Discussion}

The main pathological changes of KOA include articular cartilage degeneration, osteophyte formation, subchondral bone sclerosis, meniscus degeneration and synovial hyperplasia. These changes can cause knee pain, joint dysfunction, disability and reduced quality of life ${ }^{[11]}$. Articular cartilage degeneration is the main symptom of early KOA patients. The products of articular cartilage degeneration will stimulate the synovium and cause synovial inflammation, and synovitis will further aggravate articular cartilage degeneration. The increase of synovial exudation will increase the internal pressure of knee joint, affect the blood flow of bone and joint, and produce pain. A series of pathological changes may occur in the later 
stage. Rupture of synovial tissue, damage of vascular wall, local inflammatory edema, release of vasoactive substances and hypoxia can increase capillary permeability and cause edema of joints and surrounding tissues ${ }^{[12]}$. At present, there is no specific treatment method for KOA. Clinical treatment of the disease mainly aims at reducing pain, improving joint mobility, reducing dysfunction and improving quality of life [13].

The main component of sodium aescinate tablets is triterpenoid saponin sodium salt containing ester bond. Its precursor aescin is extracted from the mature seed of aescinate. Its pharmacological effects are [14]:

(1) anti-inflammation, detumescence and anti-exudation

(2) improving microcirculation, and enhancing vascular tension as well as contractility

(3) antioxidant and anti-free radical

(4) anti-tumor

(5) inhibit apoptosis of small intestinal tissue cells and reduce intestinal injury during I / R

(6) analgesic and neuroprotective effects. In this study, sodium aescinate tablets were applied to the treatment of KOA combined with synovitis.

The results showed that the intervention group was better than the control group in alleviating knee pain, improving joint function and improving clinical efficacy, and the difference was statistically significant. Sodium aescinate can inhibit the expression of inflammatory cytokines and has significant antiinflammatory effect ${ }^{[15-17]}$, which can first reduce the local inflammation of patients with KOA combined with synovitis; second, because it can reduce capillary permeability and improve the elasticity and tension of vascular wall ${ }^{[18-19]}$, it can reduce the edema caused by synovitis; at the same time, because it has obvious analgesic effect ${ }^{[20]}$, it can also effectively alleviate the pain caused by inflammation and swelling.

In conclusion, through the clinical control study of 86 patients with KOA combined with synovitis, the author believes that sodium aescinate tablet is an effective anti-inflammatory, anti-exudative and detumescent drug. It has good curative effect on the treatment of patients with KOA combined with synovitis and is worthy of clinical promotion.

\section{Disclosure statement}

The authors declare no conflict of interest.

\section{References}

[1] Zhang D, Wang Q, Shi X, et al., 2016, Analysis and Comparison about Musculoskeletal Ultrasonolranhv and X-rav of Knee Osteoarthritis. Chin J Othop Traumatol, 29(5): 429-433.

[2] Snchez-Romero EA, Pecos-Martn D, Calvo-Lobo C, et al., 2019, Clinical Features and Myofascial Pain Syndrome in Older Adults with Knee Osteoarthritis by Sex and Age Distribution: A CrossSectional Study. Knee, 26(1): 165-173.

[3] Tang X, Wang S, Zhan S, et al., 2016, The Prevalence of Symptomatic Knee Osteoarthritis in China: Results from the China Health and Retirement Longitudinal Study. Arthritis Rheumatol, 68(3): 648653.

[4] Ding Y, Wang J, Du L, et al., 2013, Effect of Sodium Aescinate on Nuclear Transcription Factor and Aquaporin in Brain Tissue after Intracerebral Hemorrhage in Rats and Its Correlation Analysis. China Medical Herald, 17(22): 19-21.

[5] Wei N, Yan L, 2015, Clinical Observation of Puerarin Combined with $\beta$-Aescinate Sodium in the Treatment of Limb Edema after Hand Trauma. China Pharmacy, 2015, 20(3): 2843-285. 
[6] 2018, Joint Surgery Group, Branch of Orthopedics, Chinese Medical Association. Guidelines for the Diagnosis and Treatment of Osteoarthritis (2018 Edition). Chinese Journal of Orthopaedics, 38(12): 705-715.

[7] Zhang Z, Yang Z, 2001, Orthopaedic Tendinocyst Disease. Zhengzhou: Henan Medical University Press, 213-214.

[8] Tan G, 2012, Pain Diagnosis and Treatment. Third edition Beijing: People's Medical Publishing House, 108-109.

[9] Bellamy N, Buchanan WW, Goldsmith CH, et al., 1988, Validation of WOMAC: A Health Status Instrument for Measuring Clinically Important Patient Relevant Outcomes to Anti-Rheumatic Drug Therapy in Patients with Osteoarthritis of The Hip or Knee. J Rheumatol, 15(12): 1833-1840.

[10] Zheng X, 2002, Trial Implementation of Guiding Principles for Clinical Research of New Traditional Chinese Medicine. Beijing: China Medical Science Press, 352-353.

[11] Chen D, Shen J, Zhao W, et al., 2017, Osteoarthritis: Toward a Comprehensive Understanding of Pathological Mechanism. Bone Res, 5(1): 16044.

[12] Jung K, Lee S, Hwang SH, et a1., 2010, Hish Frequency of Meniscal Hypertrophy in Persons with Advanced Varus Knee Osteoarthritis. Rheumatol Int, 30(10): 1325-1333.

[13] Hussain SM, Neilly DW, Baliga S, et al., 2016, Knee Osteoarthritis: A Review of Management Options. Scott Med J, 61(1):7- 16.

[14] Xiong Y, Li H, Peng Y, et al., 2016, Research Progress of Aescinate Saponins from Suoluo Fruit. Journal of Chinese Medicinal Materials, 39(5): 1195-1199.

[15] Selvakumar GP, Janakiraman U, Essa MM, et al., 2014, Escin At-Tenuates Behavioral Impairments, Oxidative Stress and Inflammation in a Chronic MPTP/Probenecid Mouse Model of Parkinson's Disease. Brain Research, 1585: 23-36.

[16] Lv J, Sui H, Yan F, et al., 2014, Effects of Sodium Aescinate on ET and CGRP of Cerebral Ischemiareperfusion Injury in Gerbils. Chinese Pharmacist, 17(4): 596-598.

[17] Li H, Wang J, Gao Z, 2015, Effects of Lysine Hydrochloride and Sodium Aescinate on Expression of TNF- $\alpha$ and IL- 6 in Cerebral Hemorrhage Rats. Chinese Archives of Traditional Chinese Medicine, 33(3): 688-690.

[18] Wang B, Yang R, Ju Q, et al., 2016, Clinical Effects of Joint Application of $\beta$-Sodium Aescinate and Mannitol in Treating Early Swelling after Upper Limb Trauma Surgery. Experimental \& Therapeutic Medicine, 12(5): 3320-3322.

[19] Xue X, Li Z, Zhang C, et al., 2019, Preparation of Aescinate Saponins and Its Therapeutic Effect on Hind Limb Edema in Rats. Journal of Molecular Imaging, 42(1): 46-51.

[20] Li Q, Ouyang H, Wang P, et al., 2012, The Antinociceptive Effect of Intrathecal Escin in the Rat Formalin Test. European Journal of Pharmacology, 674(2-3):234-238. 